\title{
Sexual Conflict: Mechanisms and Emerging Themes in Resistance Biology*
}

\section{Tracey Chapman ${ }^{\dagger}$}

School of Biological Sciences, University of East Anglia, Norwich Research Park, Norwich NR4 7TJ, United Kingdom Submitted October 19, 2017; Accepted March 15, 2018; Electronically published May 24, 2018

\begin{abstract}
Aвstract: Sexual conflict is acknowledged as pervasive, with the potential to generate and maintain genetic variation. Mechanistic studies of conflict have been important in providing direct evidence for the existence of sexual conflict. They have also led to the growing realization that there is a striking phenotypic diversity of adaptations whose evolution can be shaped by sexually antagonistic selection. The mechanisms involved range from the use of genital spines, claspers, songs, and smells to ejaculate molecules. In one well-studied example, sexual conflict can occur over the sexually antagonistic effects of seminal fluid proteins in Drosophila melanogaster. However, an important puzzle remains, namely, why seminal fluid proteins are so numerous and complex, hence whether all or some are involved in mediating sexual conflict. I hypothesize that this rich diversity and the complexity of traits subject to sexually antagonistic selection in general may arise, at least in part, due to the deployment of sexually antagonistic adaptations in males in a way that lessens the probability of broadscale, strong resistance evolution in females. In elaborating this hypothesis, I explore how research into the evolution of resistance to insecticides, antimicrobials, and vaccines might be used to provide insights into the evolution of female resistance to the effects of sexually antagonistic manipulative traits of males. In this manner, the manipulative traits of males can be resistance-proofed.
\end{abstract}

Keywords: sexually antagonistic selection, resistance strategies, resistance management, insecticide, antimicrobial.

\section{Introduction}

The interests of males and females (Parker 1979), or of male and female sex functions within hermaphrodites (Charnov 1979), may often diverge. This results in a pervasive opportunity for sexual conflict across a huge range of taxa (Partridge and Hurst 1998; Arnqvist and Rowe 2005; Chapman

\footnotetext{
* These articles originated as the 2017 Vice Presidential Symposium presented at the annual meetings of the American Society of Naturalists in Portland, Oregon.

${ }^{\dagger}$ Email: tracey.chapman@uea.ac.uk.

ORCIDs: Chapman, http://orcid.org/0000-0002-2401-8120.

Am. Nat. 2018. Vol. 192, pp. 000-000. (C) 2018 by The University of Chicago. 0003-0147/2018/19202-58027\$15.00. All rights reserved. DOI: $10.1086 / 698169$
}

2009). Sexual conflict was originally defined as "a conflict between the evolutionary interests of the two sexes" (Parker 1979), which was broadened to "sexually antagonistic selection on shared traits" (Rowe and Day 2006) to encompass sexual conflict mediated by the same or different loci in each sex or sex function. The evolutionary tension between the fitness interests of the different parties involved can select for strategies in which one party can gain, regardless of the expression of costs in the other (Parker 1979; Rice 1992, 1996, 1998; Rice and Holland 1997; Holland and Rice 1998, 1999; Chapman et al. 2003a; Chapman 2006; Rice et al. 2006). The resulting adaptation in one sex followed by counteradaptation in the other (Rice 1992; Arnqvist and Rowe $2002 a$ ) can act as an important driver for generating evolutionary change and maintaining genetic variation (Rice 2000; Chippindale et al. 2001; Fiumera et al. 2006). The ultimate significance of sexual conflict is its potential to lead to divergence within and between species (Chapman and Partridge 1996; Parker and Partridge 1998; Arnqvist et al. 2000; Gavrilets 2000; Gavrilets et al. 2001; Kokko et al. 2002; Kokko and Rankin 2006; Hayashi et al. 2007).

The evidence in support of sexual conflict comes from many different sources (Arnqvist and Rowe 2005) and is only briefly summarized here. An important principle is that evidence for the existence of sexual conflict requires comprehensive data on the fitness effects of the sexually antagonistic trait on both sexes (Rowe and Day 2006). From experimental evolution studies there is strong evidence of selection and counterselection in males and females arising from sexual conflict (Rice 1992) and of the fitness consequences of the arrest of evolutionary processes in one sex but not the other (Rice 1996). Several studies have also documented the existence of genetic variation in, and the evolution of, female resistance to sexually antagonistic adaptations of males (Chippindale et al. 2001; Wigby and Chapman 2004; Brommer et al. 2012). Studies in which genetic variation is partitioned between individuals has also highlighted the existence of abundant sexually antagonistic genetic variation, with the potential to result in significant in- 
tralocus sexual conflict (Chippindale et al. 2001; Foerster et al. 2007; Brommer et al. 2012). Investigation of the molecular evolution and reproductive compatibilities of reproductive proteins (Swanson and Vacquier 1995, 1998, 2002; Swanson et al. 2001a, 2001b, 2003; Levitan and Ferrell 2006; Hart et al. 2014) also shows several features of interest, including positive selection and evidence that the interactions between male and female components can be shaped by antagonistic effects (e.g., Levitan and Ferrell 2006). Evidence for coevolution between male and female reproductive proteins has also been shown (Clark et al. 2009), though the extent to which this is driven by sexually antagonistic coevolution is not yet clear.

Economic studies of the costs and benefits have been conducted across a huge range of taxa (Fricke et al. 2009) and have demonstrated many examples of adaptations in one sex that increase sex-specific fitness and simultaneously decrease it in the other (Arnqvist and Rowe 2005). When combined with experimental manipulations (Fowler and Partridge 1989; Chapman et al. 1993, 1995; Arnqvist and Rowe 1995), this has proven a valuable source of evidence for sexual conflict. Comparative analyses across species and populations of the balance between the relative and absolute levels of armaments and defenses in each sex have also revealed a fascinating landscape of finely balanced trait expression in males versus females (Arnqvist and Rowe 2002a, 2002b). When combined with experimental approaches, comparative analyses can be extremely powerful (Rowe and Arnqvist 2002; Rowe and Day 2006).

In contrast, the outcomes of comparisons between crosses between different populations has provided equivocal support for conflict (Rowe et al. 2003; Long et al. 2006). However, such studies have revealed the existence of abundant male $x$ female interaction effects (Clark et al. 1999; Arbuthnott et al. 2014), though the ultimate significance of this phenomenon is not yet wholly apparent. The outcomes of sexual conflict in terms of the resulting population dynamics may be varied and can exhibit various forms of escalation or diminution (Hayashi et al. 2007). Specific outcomes of conflict are also predicted by the "chase away" model of sexual conflict, which suggests that manipulation by males should lead to the evolution of resistance in females or the dampening down of female responses (Holland and Rice 1998; Sakaluk et al. 2006).

\section{Insights into Sexual Conflict Arising from Mechanistic Information}

Mechanistic approaches to the study of sexual conflict, on which I focus in this article, can give valuable insights. These are defined here as an understanding of how causative traits, underlying genes, or molecular pathways can influence the expression of sexual conflict. Understanding mechanisms can be important because features such as the means by which females respond to males (e.g., quantitatively or as a function of a threshold) can affect whether the outcome of sexual conflict is likely to lead to escalation or, instead, female insensitivity to the male trait (Rowe et al. 2005). In this manner, mechanistic information not only provides greater detail about the specific system under study but can also reveal the ultimate significance of the processes involved. This idea is explored further below, with a primary focus throughout on interlocus sexual conflict mediated by the effects of different genes in each sex.

Mechanistic information arising from molecular evolution studies and experimental manipulations has been key to providing evidence for sexual conflict and reveals that there is an abundance of different kinds of traits, from the molecular to the morphological, that can be shaped by sexually antagonistic selection. Mechanistic studies can also be useful for giving insights into potentially hidden processes. For example, studies of seminal fluids provide evidence for the existence of previously covert conflicts between males and females (Chapman et al. 1995; Swanson et al. 2001b, 2003; Clark et al. 2009).

An understanding of mechanistic information can also be key to predicting whether conflicts are likely to be perpetuated over time and if so, what type of population dynamics they might then assume. The importance of understanding female responses in particular (e.g., threshold or quantitative) seems key here. For example, if female resistance to male sexually antagonistic adaptations is a threshold trait (i.e., the female response is "on" or "off" above or below a certain stimulus, respectively), the potential effect of the evolution of female resistance on male fitness is greater than if the female resistance trait evolves to change its sensitivity (i.e., via a quantitative response; Rowe and Day 2006). This effect arises because the threshold versus sensitivity responses of females impose different fitness consequences for males. Hence, the fitness costs depend on the mechanistic basis of the female trait (threshold or sensitivity) and the strength of selection on both sexes. Arnqvist (2006) illustrated an example of how a quantitative female trait subject to sexual conflict could be manipulated. In this scenario, female responses to males show a simple dose response to the level of a gonadotropin, and an intermediate, optimal dose maximizes female fitness. Under polyandry, males have less interest in their current partner's future reproduction, and hence males that can exploit mechanisms in females to elevate the level of gonadotropin gain fitness, but females suffer costs because they are shifted off their optimum (Arnqvist 2006).

Mechanistic information can also be key in understanding the likely outcomes of sexually antagonistic competitions between different males mating with the same female. For example, the outcomes of sperm competition may depend on whether the degree of sperm competition perceived or experienced coevolves with or is independent from sperm 
allocation (Williams et al. 2005). Similarly, theory shows that insight into the mechanisms by which fertilization bias is achieved (effect of sperm vs. nonsperm components) is essential to gain an understanding of how ejaculates will evolve (Cameron et al. 2007). The extent of natural selection on the female sensory system potentially involved in sexually antagonistic interactions can also determine the outcome of sexual conflict - specifically, whether this is likely to lead to an arms race or alternatively to a dampening down of conflict via the evolution of female insensitivity (Rowe et al. 2005). The extent of female costs is a crucial parameter in this context. The flexibility of female preference (e.g., the extent to which females that have greater sensory information have greater flexibility to inform their preferences) is central to understanding the outcome of sexual conflict (Rowe et al. 2005) but is as yet poorly understood. An emerging theme from these studies is that the female responses are important in determining whether a conflict will be escalated or diminished. It is the way in which females respond that determines whether resistance to any manipulative effects of males can occur and if so, how rapidly or in which direction.

\section{A Mechanistic Case Study of Sexual Conflict in Drosophila melanogaster}

There is good evidence for sexual conflict resulting from the actions of seminal fluid proteins in Drosophila melanogaster. Evidence for the involvement of seminal fluid proteins in mediating costs of mating (Fowler and Partridge 1989) first emerged from findings such as mating costs not being reduced in matings with spermless males (Chapman 1992; Chapman et al. 1993). Later manipulations using males in which the main secretory cells of the male accessory gland were genetically ablated pinpointed the fact that the seminal fluid proteins of the male were responsible for causing female mating costs in an apparently dose-dependent manner (Chapman et al. 1995). The seminal fluid proteins (Sfps) involved were well known to be involved in mediating a range of potentially costly postmating responses, including sperm competition. This gave rise to the hypothesis that the Sfps functioning in males to ensure high per mating reproductive success were somehow costly to females as a byproduct. Hence, Sfps could mediate sexual conflicts between the evolutionary interests of males (namely, to increase their per mating reproductive success) and the longer-term reproductive interests of females (via higher reproductive success through mating less). Later work then focused on whether particular Sfps were responsible. The well-characterized sex peptide (Chen et al. 1988) was noted as an early candidate from a study that indicated that a lack of response to sex peptide could increase mating costs (Chapman et al. 1996). Matings with males lacking sex peptide also appeared to be cost free (Wigby and Chapman 2005). However, variation in the nutritional environment, and presumably the ability to express the sex peptide's effects, can alter whether sex peptide receipt is costly or beneficial to females (Fricke et al. 2010). Other research, using ectopic expression of additional Sfps, including sex peptide, also supported the idea that exposure to Sfps can be costly to females (Chapman et al. 1995; Lung et al. 2002; Mueller et al. 2007).

Perhaps the defining feature of Sfps in D. melanogaster, including those so far implicated in mediating sexual conflict, is their mechanistic complexity (Ram and Wolfner 2007a, 2009; Sirot et al. 2014). For example, well over 120 seminal fluid proteins, with hugely diverse effects on female behavior and physiology, are transferred to females during mating (Findlay et al. 2008; fig. 1). As noted above, the receipt of these proteins, and of some in particular such as the sex peptide, can result in the expression of costs in females (Chapman 1992; Chapman et al. 1993, 1995, 1996; Lung et al. 2002; Wigby and Chapman 2005; Mueller et al. 2007; Fricke et al. 2010). Whether the receipt of one, some, or all Sfps can be costly to females is not yet clear. What is intriguing is the possibility that the seminal fluid appears, from some perspectives, as "overly complicated."

Sfps fall into several functional classes, with multiple examples of Sfps having putative roles in carbohydrate binding, chitin binding, defense/immunity, and proteases, protease inhibitors, lectins, lipases, cysteine-rich secretory proteins (CRISPs), and odorant-binding proteins, as well as many Sfps with as yet unidentified functions (Findlay et al. 2008). There is the potential for considerable redundancy within these classes. For example, there are at least 15 proteases, 14 protease inhibitors, 12 involved in defense/immunity, nine involved in lipid metabolism, and seven involved in odorant binding (Findlay et al. 2008). At least five Sfps are known to have effects on female receptivity, and six are known to have effects on egg production (Ram and Wolfner 2009). However, multiple members of the same class may also have distinct roles in fine-tuning the same type of responses (Wolfner 1997, 2002; Ram and Wolfner 2007a). It has been suggested that the redundant complexity of Sfps could reveal evidence for past sexual conflicts (Tregenza et al. 2006), with relics of former players in conflicts retained and giving the pattern of redundancy. This is not yet proven, but such evidence could emerge if there are Sfps within each functional class with null effects.

Ultimately, discovery of the distribution of phenotypic effects of Sfps subject to sexually antagonistic selection will be of great interest here. Of those Sfps that are involved, it will be important to ascertain whether they have single or multiple effects and if the latter, whether their effects are linked or independent. If Sfps subject to conflict have multiple effects, then it is also possible that they target multiple, even predictable, pathways in females. 


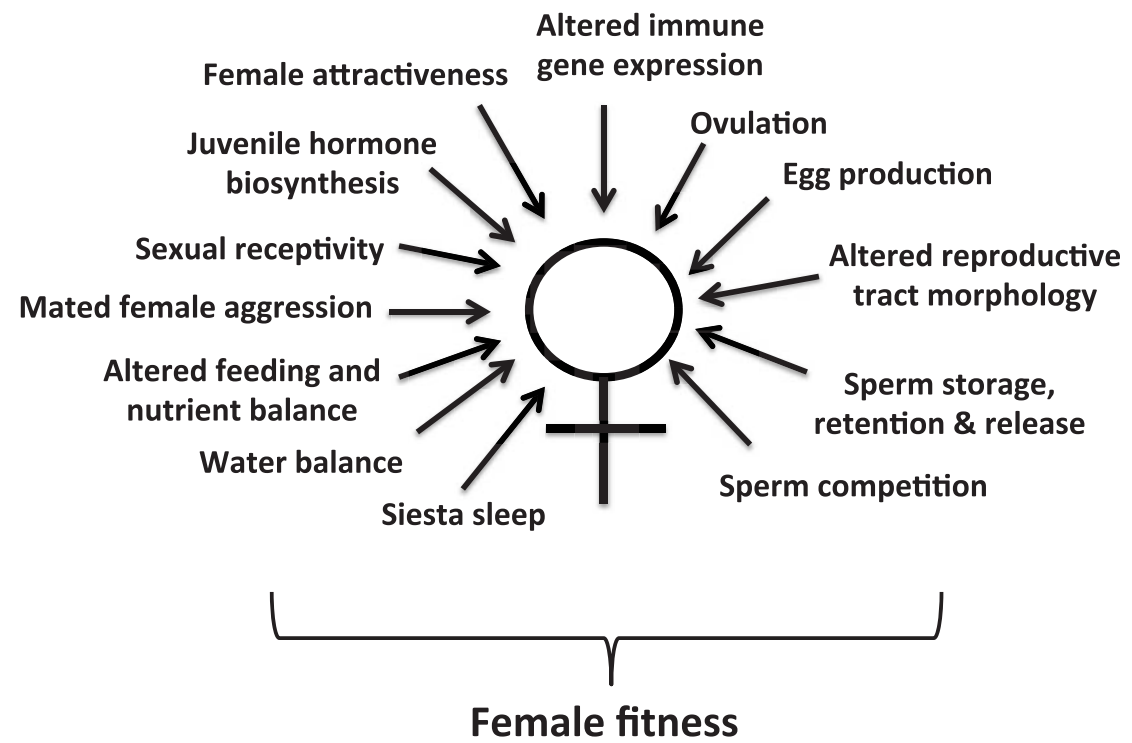

Figure 1: Seminal fluid proteins (Sfps) cause a profound remodeling of female behavior and physiology. Individual Sfps or sets of Sfps can reduce female sexual receptivity (Chapman et al. 2003b; Liu and Kubli 2003; Bretman et al. 2009); affect female attractiveness (Tram and Wolfner 1998); reduce siesta sleep (Isaac et al. 2009); increase feeding (Carvalho et al. 2006); alter nutrient balancing (Ribeiro and Dickson 2010) and water balance (Cognigni et al. 2011); increase female aggressive behavior following mating (Bath et al. 2017); increase female attractiveness (Tram and Wolfner 1998); increase ovulation (Heifetz et al. 2000,2005) and egg production (Chapman et al. 2003b; Liu and Kubli 2003; Ram and Wolfner 2009); increase the synthesis of juvenile hormone from the corpus allatum (Moshitzky et al. 1996); alter the expression of immune genes (Peng et al. 2005; Domanitskaya et al. 2007) and potential immunity (Lung and Wolfner 2001); affect the storage, retention, and release of sperm in storage (Neubaum and Wolfner 1999; Tram and Wolfner 1999; Wong et al. 2008; Avila et al. 2010); alter the conformation of the female reproductive tract following mating (Avila and Wolfner 2009); and finally, affect the outcome of sperm competition (Fiumera et al. 2005, 2007; Mueller et al. 2008; Chow et al. 2010; Smith et al. 2017). The actions of seminal fluid proteins are reported to decrease female fitness (Chapman et al. 1995; Lung et al. 2002; Mueller et al. 2007) in a manner that is dependent on the environment and hence the ability to express sexually antagonistic adaptations (Fricke et al. 2010).

With regard to the distribution of functions across Sfps, the evidence so far suggests that some Sfps (such as sex peptide) may do several jobs and others fewer. Whether sex peptide is a typical example of one or several master regulators will be interesting to discover. Sex peptide is interesting because it is a single Sfp whose actions are subject to sexual conflict that has different effects on a range of different traits in females: sexual receptivity and egg production (Chapman et al. 2003b; Liu and Kubli 2003), siesta sleep (Isaac et al. 2009), water balance (Cognigni et al. 2011), female aggression (Bath et al. 2017), feeding (Carvalho et al. 2006), nutrient balancing (Ribeiro and Dickson 2010), and the release of sperm from storage (Avila et al. 2010). Sex peptide also interacts with other Sfps in maintaining longer-term postmating responses (Ram and Wolfner 2007b, 2009). It is not yet fully evident whether the multiple phenotypes influenced by sex peptide each operate fully independently from one another. The potential for this to be the case is suggested by work that shows that although sex peptide responses in egg production and female receptivity are usually linked (Chapman et al. 2003b; Liu and Kubli 2003), they can be uncoupled in females of different ages (Fricke et al. 2013). It is also not yet clear the extent to which the varied effects of one Sfp, such as sex peptide, can proceed in isolation of the effects of other Sfps, though it is known that a network of other Sfps are needed for sex peptide to interact normally with sperm (Ram and Wolfner 2009). Hence, it is possible that the sex peptide targets multiple, potentially independent pathways in females.

The hypothesis explored below is that this reflects the actions of selection on males to employ a strategy of manipulation to slow the evolution of female resistance. I investigate the possibility that a single Sfp, such as sex peptide, with multiple independent effects (a "master regulator"; Gioti et al. 2012) can slow the evolution of resistance in females more effectively than is the case for individual Sfps, each targeting single, independent effects.

\section{Male Manipulation Strategies and the Evolution of Female Resistance-Employing Principles from the Evolution of Resistance against Insecticides and Antimicrobials}

As outlined in the previous section, the distribution of phenotypic effects of Sfps subject to sexually antagonistic selec- 
tion is likely to be fundamental to the ease and speed of female resistance evolution. In the rest of this article, it is this question that is considered-specifically, whether using principles of resistance management gained from the study of insecticides and antimicrobials can explain the way in which manipulative strategies are deployed. Two hypotheses are explored:

Hypothesis 1. The deployment of sexually antagonistic adaptations in males occurs in a way that lessens the evolution of broad-scale resistance in females.

Hypothesis 2. The benefits of deploying multiple manipulation mechanisms explains the rich diversity of different kinds of adaptations that become subject to sexually antagonistic selection.

There is a long history of the study of resistance in the face of selection arising from insecticide application and the widespread use of antimicrobials (table 1). However, until recently there has been relatively little cross talk between these two fields of study or with the evolutionary study of vaccination (REX Consortium 2010). There are four types of strategies generally employed by humans to slow the evolution of insecticide or antimicrobial resistance (table 1). Under "responsive alternation," a strategy is employed until resistance becomes apparent and it is then replaced with another, to which there might be only a low level of resistance. Theory and empirical studies suggest that this strategy is generally the least effective in slowing resistance (REX Consortium 2012) because it necessarily allows the emergence of resistance genes and then requires an alternative strategy. Applying this to the scenario of sexually antagonistic selection (table 1 ) is akin to a single manipulative strategy selecting for resistance in females. Interestingly, this form of selection of sexually antagonistic alleles has been shown by Mokkonen et al. (2011) in the context of intralocus conflict. The "periodic application" strategy (Coyne 1951 ) is similar to responsive alternation, except the cycling is regular and occurs if two or more different strategies are deployed in a regular, cyclical manner, likely prior to the emergence of resistance. It is thought to be slightly more effective than responsive alternation because it does not select so strongly for resistance or at least does not allow its emergence to the same degree before moving to an alternative strategy. However, it is not straightforward to envisage any natural scenario in which this could apply to sexual conflict, due to the absence of an external organizing agent (table 1). In both the responsive alternation and periodic strategies, the deployment of mechanisms varies through time but not space. The long-term cycling common to both strategies is predicted to be unlikely to prevent the evolution of resistance (Bergstrom et al. 2004).

"Mosaic" is a strategy that varies across space, with different strategies being used in different places, but not time (table 1). Along with periodic application, this strategy is better able to slow resistance than is responsive alternation (REX Consortium 2012). In terms of sexual conflict, this could represent a situation where males in different demes or populations employ distinct manipulative mechanisms, a scenario that could in principle occur in natural situations. The final strategy is "combination" (Curtis 1985), and in this, different agents are employed simultaneously. The evidence suggests that combination strategies are least likely, in comparison to all others, to lead to resistance.

Combination appears to work best because individuals are unlikely to carry multiple resistance alleles. Hence, they can be targeted effectively by at least one of the agents being used (a phenomenon termed "multiple intergenerational killing"; table 1). A scenario in which this could occur under sexual conflict would be if individual males exhibit variation in multiple, independent mechanisms for manipulating females, with relatively limited cross talk between them. Such scenarios seem possible and may pertain to the example of seminal fluid proteins described above (figs. 1, 2) Males could vary in the extent to which they use each independent strategy to manipulate females in a manner that selects only weakly for female resistance. A pattern resembling combination could also arise if males within the same population employed different, independent manipulative mechanisms. However, in this case, female resistance against any one of the strategies employed by individual males could decrease the fitness of the individual male employing that mechanism to a greater extent than is likely to be the case for individual males that can express multiple mechanisms. Combinatorial strategies in pest and microbe resistance have also been explored in both full- or low-dose scenarios, and this idea is explored further below.

In resistance management, the concept of treatment heterogeneity is also key, with the evolution of resistance being harder to evolve when this is high (Mani 1989). Hence, if there is the equivalent of high-treatment heterogeneity, that is, wide variation in the deployment of resistance strategies among males, the evolution of resistance would be slowed due to the likelihood that some of the strategies would always be effective, even if some were not (table 1). In the two theoretical scenarios illustrated in figure 2, females are exposed to varying strengths of multiple manipulative adaptations from either the same male or different males. The fitness consequences for males are likely to vary because in the former scenario males can deploy a combination of manipulations in concert, weakening selection for resistance. There is growing evidence of significant genetic variation among females in responses to male traits that are subject 


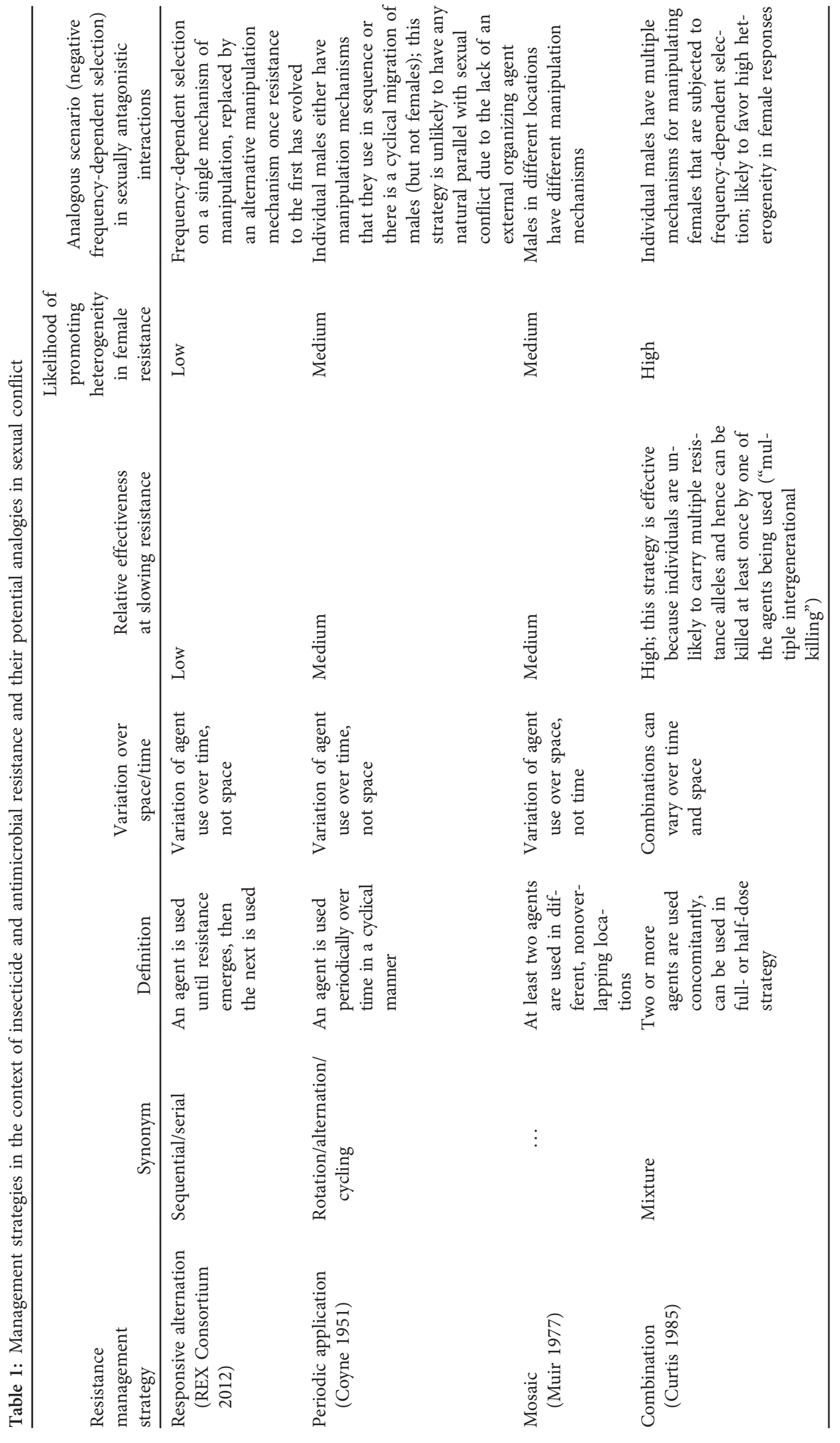

This content downloaded from 139.222.162.213 on May 31, 2018 01:52:13 AM 
(a)
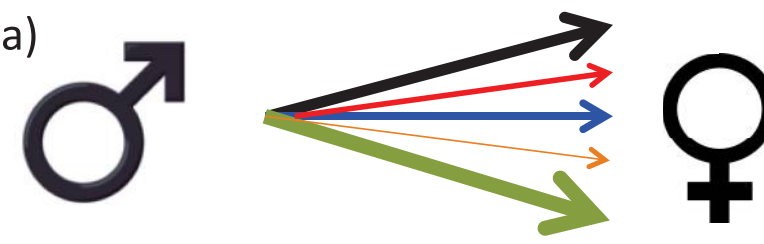

(b)

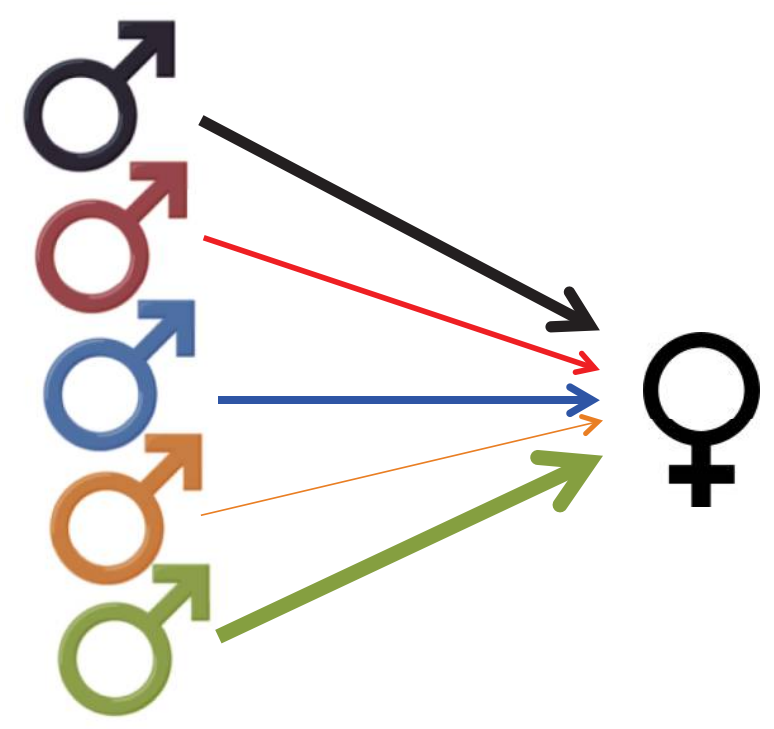

Figure 2: Contrasting male manipulation strategies. $a$, A strategy generalist. Individual males deploy varying strengths (arrow widths) of multiple, qualitatively different and potentially independent manipulative adaptations (colors). The different strategies deployed by individual males could have fixed or flexible strengths. $b$, A strategy specialist. Different males (colors) deploy varying strength of qualitatively different (arrow widths), potentially independent manipulative adaptations. Again, the different strategies deployed by different males could have fixed or flexible strengths. The fitness consequences for individual males are likely to differ significantly. In $a$, males gain from some of their manipulative strategies even if there is female resistance to others. Overall selection for female resistance is weak. In $b$, some males gain by using manipulations to which there is no resistance and others do not. Selection for resistance is related to the frequency of each strategy in the population and is generally expected to be stronger due to the lack of multigenerational killing (table 1).

to sexual conflict (Wigby and Chapman 2004; Linder and Rice 2005). However, exactly how this variation is distributed through time and space remains to be determined, as does the extent to which female responses and male traits share significant genetic correlations.

Strategies of resistance management involving rotations and mixtures can also be undermined by cross-resistance of the selective agent. In a sexual conflict perspective, a male would then gain from this type of strategy only if (i) there are multiple routes for manipulating females that are relatively independent and (ii) there is variation in the usage of different mechanisms across males. It is not yet clear whether the first condition is fulfilled, but there are examples of evidence for significant genetic variation in males for traits subject to sexual conflict (e.g., Smith et al. 2009).

Combination is predicted to work best at slowing resistance evolution, except under scenarios in which there are significant fitness costs of being resistant (expressed in the absence of the agent; REX Consortium 2012). In strict combination scenarios, the selective agent is never absent. Hence, fitness costs are only minimally expressed and do not contribute to resistance evolution. The existence of costs of resistance could delay the evolution of resistance in strategies in which the selective agents are rotated or sometimes absent or in environments in which there are refuges or in which compensatory mutations evolve (e.g., Guillemaud et al. 1998; Paris et al. 2008; Andersson and Hughes 2010). The idea that fitness costs are important has also been explored in theory that suggests that it is not the strength of selection that is a primary determinant of the rate of resistance evolution but instead the absolute fitness of the resistant forms (rather than their relative fitness in comparison to sensitive strains; Day et al. 2015). These authors also emphasize the importance of competitive release (i.e., removal of the selective agent) in the evolution of resistance.

It is clear that making analogies between humanmanaged resistance and male resistance strategies within sexual conflict has some limitations. For example, there are some types of resistance strategies, such as periodic application (table 1), that can have no analogy in sexual conflict due to the absence of an external organizing force. There are also contrasts between the nature of selection, for example, that imposed by the population structure of genes subject to sexual conflict versus treatment regimes imposed over spatial and temporal scales for insecticides or antimicrobials (meaning that individuals could be selected in one generation but not the next - a scenario that seems unlikely under sexual conflict). However, it is also possible that strategies that resemble combinations in which pesticide or antimicrobial resistance is observed to be slow could be selected in males precisely for those reasons and in the absence of any external organizer. It is the properties of these strategies that are worthy of further examination. For example, the application of these ideas suggests the possibility that many male traits might evolve to accrue short-term benefits but that resistance would quickly evolve to them. Hence, the more persistent pathways exploited by males could be those that exhibit features of combination strategies (table 1), and these would evolve along such paths where resistance cannot evolve. 


\section{"Hit Hard" versus Lower-Dose-Perfect versus Imperfect Strategies}

The question of whether it is best to hit hard in the management of insecticide or antimicrobial resistance evolution remains controversial. On the one hand, if an agent is applied so forcefully that all insects or microbes are killed, then resistance is never likely to evolve, as there are no individuals that survive following exposure to the selective agent. However, should just a few escape, they have a huge selective advantage and their resistance genes will rapidly spread, quickly negating the benefits of the initial hit-hard strategy. A similar concern pertains to so-called leaky vaccines, that is, those that allow the host to survive and that do not prevent the transmission of the pathogen. Theory and experiments show that this can allow the evolution of more potent pathogens (Read et al. 2015). High-dose chemotherapy may suffer from the same problem (Day and Read 2016) because of the mutational input of resistant strains and their release from competition leading to a direct relationship between selection and resistance.

For these reasons, the benefits of hit-hard strategies employing maximum doses carry huge risks. For selection to decrease the probability of the evolution of resistance, the optimum may be for selection to be either very weak (to deliver the "minimum effective") or very strong (the "maximum tolerated"; Day and Read 2016) but with the caveat that under very strong selection, escapes must be avoided. Intermediate levels of selection seem worst in terms of favoring resistance, but which strategy is best seems to vary in a manner that is not yet fully understood.

Following this general line, Read et al. (2009), working on malaria, have asked whether it is possible to create a strategy in which resistance would never evolve. Their answer is yes, by targeting the malaria parasites that would be transmitted to humans, not necessarily by killing the mosquito vectors. Their strategy is built on a mechanistic understanding of the system, recognizing that the majority of mosquitoes are not the problem and die before transmitting malaria. Hence, they propose that if insecticides could be designed to kill only older mosquitoes, resistance to antimalarials could be halted.

Collectively, the findings of these studies support the idea that there are better ways than the hit-hard strategy by which to retard the evolution of resistance (Day and Read 2016) and that strategies of delayed action activity may ultimately be more successful. This is interesting to consider here in the context of sexually antagonistic selection. The analogy would suggest that males will have to tread a difficult line: they need to deliver the minimum required level of input(s) to activate reproductive processes in femalesbenefiting both sexes. They may then gain additional, sexspecific benefits by pushing female responses higher, but if they push too hard, they may select too strongly on female resistance and generate "super-resistant" females that are insensitive to male manipulations (Rowe et al. 2005). If males manage to impose no more selection than needed to gain benefits from sexually antagonistic adaptations, they may be able to avoid the problem of so-called radical pathogen cure for increased resistance in females, that is, the inadvertent creation, via rapid removal of the selective agent, of an environment that actually favors the resistant individuals (Read et al. 2011). Hence, males that manipulate only to the extent that has the desired effect but no more, in effect managing the degree of manipulation, might gain due to the slowing of female resistance. A determination of the spectrum of how selection relates to the likelihood of resistance for variable numbers of different manipulation pathways and different resistance costs would be very useful.

\section{Female Responses to Sexually Antagonistic Manipulation}

The main focus has been on the strategies potentially used by males to slow resistance evolution rather than the nature of the female responses themselves. However, though the mechanisms underlying female responses are understudied, they are crucially important in predicting the likely escalation or diminution of conflict. It has been observed that there may be substantial constraints on female responses due to males employing mechanisms to exploit perceptual biases (Arnqvist 2006; Ryan and Cummings 2013), placing females in a "sensory trap" (West-Eberhard 1979). For example, a lack of response by females to male mating signals would lead to a failure to reproduce successfully. Hence, some level of female response to potentially harmful and manipulative signals needs to be retained. This indicates the existence of an underlying asymmetry in the extent to which females may benefit from responding versus the extent to which males benefit from the multiple manipulative mechanisms they may deploy. However, it has been suggested that perceptual biases of females may exact fewer costs than expected if they also generate direct benefits (Ryan and Cummings 2013), which could outweigh indirect benefits (Kirkpatrick and Barton 1997).

In a broader context, it would be interesting to study whether the responses of females exhibit features analogous to "pyramiding," that is, the combining of resistance genes within individuals. In the study of insecticide and antimicrobial resistance, this strategy is observed to result in a more durable strategy of resistance (REX Consortium 2016). In this way, females could be selected to retain multiple pathways of potential resistance to male manipulative strategies. Related to this, there are important facets of the mating system and interaction of females with social and environ- 
mental variation that will shape the evolution of female resistance. For example, if females routinely mate with more than one male (e.g., Imhof et al. 1998), they may need to counter several different male manipulation strategies (fig. 2). This is particularly so if males exhibit specialism or generalism in their manipulative strategies or facultative variation between the two (fig. 2). Under this scenario, selection for specialist versus generalist manipulators and responders would also show sex-specific variation. If females generally mate with several males, they will benefit from generalist resistance strategies. However, many males may mate with only one female, and many may not mate at all, potentially giving higher benefits for specialist manipulators. A corollary of this is that in each generation, females may encounter the manipulative strategies of only a subset of the males present in a population. Many strategies are also contained within males that do not mate but that could in principle be reassembled via recombination in relatives via future generations. The potential influence of these asymmetries and of gene flow and spatial heterogeneity on the likelihood and speed of female resistance evolution requires further study.

\section{Future Directions}

To determine the likely importance of sexual conflict as a force for driving evolutionary change in nature, there is a pressing need to better understand, with greater resolution, the distribution of sexually antagonistic genetic variation across time and space. For example, to fully test whether sex peptide phenotypes have evolved in a manner reminiscent of any of the strategies described in table 1 requires an improved understanding of the distribution of genetic variation underpinning sex peptide effects and female responses to it. That such variation exists is amply shown by studies that reveal genetic variation in sex peptide production (Smith et al. 2009), in female responses to sex peptide (J. Rouhana, B. Wertheim, and T. Chapman, unpublished data), and in the interactions between both sexes due to this pathway (Chow et al. 2010). Fitness surfaces of sex peptide effects also suggest different routes for males to be successful (Fricke and Chapman 2017), which could promote heterogeneity in female responses. Observations of variation in the expression of mating costs across populations (Fiumera et al. 2006; Arbuthnott et al. 2014) could represent a signature of the deployment of different resistance strategies.

In general, the intersection of ecology and sexual conflict in driving divergence has been neglected (Arbuthnott et al. 2014). This is an important omission because it has been shown that both the effect of males on female life span and of female resistance to male effects can evolve alongside adaptation to different ecological environments (Arbuthnott et al. 2014; Perry et al. 2017). Hence, the evolution of sexual conflict traits can occur in parallel with ecology, leading to the interesting outcome that ecological context might predict the outcome of sexual conflict.

That there is variation in sexually antagonistic genetic variation across different populations with different ecologies is also indicated by population-specific resistance observed in studies across a number of different vertebrate and invertebrate taxa (Arnqvist and Rowe 2005; Innocenti and Morrow 2010; Khila et al. 2012; Arbuthnott et al. 2014; Lonn et al. 2017). Such information could provide evidence for whether males actually deploy resistance-slowing strategies (table 1; fig. 2) and if so, the resulting outcomes for resistance evolution. It would also be possible to take an experimental evolution approach and impose combinations of different male strategies within and across individuals in time and space. This would allow tests of the fitness benefits of different combinations of manipulative strategies to be measured directly. It is also important to investigate whether there is cross-resistance across different male manipulation mechanisms. Here, the nature of female responses is key - it could, for example, reduce the effectiveness of combination strategies that males might employ to slow resistance. Such studies could involve tests of whether females that are resistant to one sex peptide-induced phenotype are also resistant to another.

In general, we lack knowledge of the mechanisms underlying resistance to sexual conflict in females, and this represents a challenge in understanding the evolutionary outcomes of sexual conflict. For example, whether female responses are governed by thresholds or sensitivity is key to conflict resolution or escalation (Rowe et al. 2005) as well as to the identification of the full range of genetic or selective constraints (Arnold 1992; Rodriguez and Snedded 2004). It would also be interesting to probe more deeply into whether the speed of female resistance evolution is influenced by sensory context. For example, comparisons of signal magnitudes based on proportional (not absolute) differences may be useful, as there may be a slower rate of resistance evolution when sensory traits are larger or more noticeable (Ryan and Cummings 2013). Some aspects of sensory biology (e.g., Weber's law) are indistinguishable from the chase-away model of evolution in response to sexual conflict (Holland and Rice 1998), hence a greater understanding of sensory context may be useful to distinguish these different alternatives.

Ultimately, it remains to be seen whether the approach suggested here of seeking to apply principles from resistance evolution in insecticides and antimicrobials to the arena of sexual conflict will provide additional insights. If it does, then there may be additional benefits from a further extrapolation to the evolution of resistance to vaccines versus drugs (Kennedy and Read 2017). The potential interest is that many vaccines are never selected against, potentially because they work prophylactically and induce a variety of 
immune responses, which slows resistance. The study of any such analogous process, if it occurs in sexual conflict, could provide fascinating new insights.

\section{Conclusion}

In this article, I have summarized key features and the importance of sexual conflict. I have emphasized the importance of understanding mechanisms, not only for a deeper understanding but because several facets of conflicts, including their ultimate outcome, may depend on such knowledge. I suggest that there are useful insights to be gained from considering selection on the sexually antagonistic adaptations of males and female resistance to them in the context of the management of resistance by humans. The principles emerging from this work for slowing the evolution of resistance may pertain to the deployment of sexually antagonistic strategies in males. They may also help explain the distribution of sexual conflict traits and the genetic variation that underlies them.

\section{Acknowledgments}

I thank Locke Rowe for inviting me to take part in this hugely exciting and stimulating American Society of Naturalists (ASN) Vice Presidential Symposium and to all the speakers in it. I also thank Troy Day, Locke Rowe, and Jon Bridle for ideas, encouragement, and suggestions. I also acknowledge the help of two anonymous reviewers whose suggestions greatly improved this article. I thank the ASN and the University of East Anglia for funding to attend the 2017 Evolution meeting and the Biotechnology and Biological Sciences Research Council and Natural Environment Research Council (BB/L003139/1; BB/H008047/1; NE/K0046 97/1; NE/J024244/1) for financial support enabling the research that fed into this article.

\section{Literature Cited}

Andersson, D., and D. Hughes. 2010. Antibiotic resistance and its cost: is it possible to reverse resistance? Nature Reviews Microbiology 8:260-271.

Arbuthnott, D., E. M. Dutton, A. F. Agrawal, and H. D. Rundle. 2014. The ecology of sexual conflict: ecologically dependent parallel evolution of male harm and female resistance in Drosophila melanogaster. Ecology Letters 17:221-228.

Arnold, S. J. 1992. Constraints on phenotypic evolution. American Naturalist 140(suppl.):S85-S107.

Arnqvist, G. 2006. Sensory exploitation and sexual conflict. Philosophical Transactions of the Roval Society B 361:375-386.

Arnqvist, G., M. Edvardsson, U. Friberg, and T. Nilsson. 2000. Sexual conflict promotes speciation in insects. Proceedings of the National Academv of Sciences of the USA 97:10460-10464.

Arnqvist, G., and L. Rowe. 1995. Sexual conflict and arms races between the sexes: a morphological adaptation for control of mating in a female insect. Proceedings of the Royal Society of London B 261:123-127.

2002a. Antagonistic coevolution between the sexes in a group of insects. Nature 415:787-789.

. 2002b. Correlated evolution of male and female morphologies in water striders. Evolution 56:936-947.

NJ

Avila, F. W., K. Ravi Ram, M. C. Bloch Qazi, and M. F. Wolfner. 2010. Sex peptide is required for the efficient release of stored sperm in mated Drosophila females. Genetics 186:595-600.

Avila, F. W., and M. Wolfner. 2009. Acp36DE is required for uterine conformational changes in mated Drosophila females. Proceedings of the National Academy of Sciences of the USA 106:15796-15800.

Bath, E., S. Bowden, C. Peters, A. Reddy, J. A. Tobias, E. EastonCalabria, N. Sneddon, S. F. Goodwin, and S. Wigby. 2017. Sperm and sex peptide stimulate aggression in female Drosophila. Nature Ecology and Evolution 1:0154.

Bergstrom, C. T., M. Lo, and M. Lipsitch. 2004. Ecological theory suggests that antimicrobial cycling will not reduce antimicrobial resistance in hospitals. Proceedings of the National Academy of Sciences of the USA 101:13285-13290.

Bretman, A., M. K. N. Lawniczak, J. M. Boone, and T. Chapman. 2009. A mating plug protein reduces early female remating in Drosophila melanogaster. Journal of Insect Physiology 56:107-113.

Brommer, J. E., C. Fricke, D. A. Edward, and T. Chapman. 2012. In teractions between genotype and sexual conflict environment influence transgenerational fitness in Drosophila melanogaster. Evolution 66:517-531.

Cameron, E., T. Day, and L. Rowe. 2007. Sperm competition and the evolution of ejaculate composition. American Naturalist 169:E158E172.

Carvalho, G. B., P. Kapahi, D. J. Anderson, and S. Benzer. 2006. Allocrine modulation of feeding behavior by the sex peptide of Drosophila. Current Biology 16:692-696.

Chapman, T. 1992. A cost of mating with males that do not transfer sperm in female Drosophila melanogaster. Journal of Insect Physiology 38:223-227.

2006. Evolutionary conflicts of interest between males and females. Current Biology 16:744-754.

. 2009. Sexual conflict and sex allocation. Biology Letters 5:660-662

Chapman, T., G. Arnqvist, J. Bangham, and L. Rowe. 2003a. Sexual conflict. Trends in Ecology and Evolution 18:41-47.

Chapman, T., J. Bangham, G. Vinti, B. Seifried, O. Lung, M. F. Wolfner, H. K. Smith, and L. Partridge. 2003b. The sex peptide of Drosophila melanogaster: female post-mating responses analyzed by using RNA interference. Proceedings of the National Academy of Sciences of the USA 100:9923-9928.

Chapman, T., Y. Choffat, W. E. Lucas, E. Kubli, and L. Partridge. 1996. Lack of response to sex-peptide results in increased cost of mating in dunce Drosophila melanogaster females. Journal of Insect Physiology 42:1007-1015.

Chapman, T., J. Hutchings, and L. Partridge. 1993. No reduction in the cost of mating for Drosophila melanogaster females mating with spermless males. Proceedings of the Roval Society of London B 253:211-217.

Chapman, T., L. F. Liddle, J. M. Kalb, M. F. Wolfner, and L. Partridge. 1995. Cost of mating in Drosophila melanogaster females is mediated by male accessory gland products. Nature 373:241-244. 
Chapman, T., and L. Partridge. 1996. Sexual conflict as fuel for evolution. Nature 381:189-190.

Charnov, E. L. 1979. Simultaneous hermaphroditism and sexual selection. Proceedings of the National Academy of Sciences of the USA 76:2480-2484.

Chen, P. S., E. Stumm-Zollinger, T. Aigaki, J. Balmer, M. Bienz, and P. Böhlen. 1988. A male accessory gland peptide that regulates reproductive behavior of female D. melanogaster. Cell 54:291298.

Chippindale, A. K., J. R. Gibson, and W. R. Rice. 2001. Negative genetic correlation for adult fitness between sexes reveals ontogenetic conflict in Drosophila. Proceedings of the National Academy of Sciences of the USA 98:1671-1675.

Chow, C. Y., M. F. Wolfner, and A. G. Clark. 2010. The genetic basis for male $\times$ female interactions underlying variation in reproductive phenotypes of Drosophila. Genetics 186:1355-1365.

Clark, A. G., D. J. Begun, and T. Prout. 1999. Female $\times$ male interactions in Drosophila sperm competition. Science 283:217-220.

Clark, N. L., J. Gasper, M. Sekino, S. A. Springer, C. F. Aquadro, and W. J. Swanson. 2009. Coevolution of interacting fertilization proteins. PLoS Genetics 5:e1000570.

Cognigni, P., A. P. Bailey, and I. Miguel-Aliaga. 2011. Enteric neurons and systemic signals couple nutritional and reproductive status with intestinal homeostasis. Cell Metabolism 13:92-104.

Coyne, F. P. 1951. Proper use of insecticides. British Medical Journal 2:911-912.

Curtis, C. F. 1985. Theoretical models of the use of insecticide mixtures for the management of resistance. Bulletin of Entomological Research 75:137-155.

Day, T., S. Huijben, and A. F. Read. 2015. Is selection relevant in the evolutionary emergence of drug resistance? Trends in Microbiology 23:126-133.

Day, T., and A. F. Read. 2016. Does high-dose antimicrobial chemotherapy prevent the evolution of resistance? PLoS Computational Biology 12:e1004689.

Domanitskaya, E. V., H. Liu, S. Chen, and E. Kubli. 2007. The hydroxyproline motif of male sex peptide elicits the innate immune response in Drosophila females. FEBS Journal 274:5659-5668.

Findlay, G. D., X. Yi, M. J. MacCoss, and W. J. Swanson. 2008. Proteomics reveals novel Drosophila seminal fluid proteins transferred at mating. PLoS Biology 6:e178.

Fiumera, A. C., B. L. Dumont, and A. G. Clark. 2005. Sperm competitive ability in Drosophila melanogaster associated with variation in male reproductive proteins. Genetics 169:243-257.

2006. Natural variation in male-induced "cost-of-mating" and allele-specific association with male reproductive genes in Drosophila melanogaster. Philosophical Transactions of the Roval Societv B 361:355-361.

. 2007. Associations between sperm competition and natural variation in male reproductive genes on the third chromosome of Drosophila melanogaster. Genetics 176:1245-1260.

Foerster, K., T. Coulson, B. C. Sheldon, J. M. Pemberton, T. H. Clutton-Brock, and L. E. B. Kruuk. 2007. Sexually antagonistic genetic variation for fitness in red deer. Nature 447:1107-1111.

Fowler, K., and L. Partridge. 1989. A cost of mating in female fruitflies. Nature 338:760-761.

Fricke, C., A. Bretman, and T. Chapman. 2010. Female nutritional status determines the magnitude and sign of responses to a male ejaculate signal in Drosophila melanogaster. ology 23:157-165.
Fricke, C., and T. Chapman. 2017. Variation in the post-mating fitness landscape in fruitflies. Journal of Evolutionary Biology 30:1250-1261.

Fricke, C., D. Green, W. E. Mills, and T. Chapman. 2013. Age-dependent female responses to a male ejaculate signal alter demographic opportunities for selection. Proceedings of the Roval Societv B 280: 20130428.

Fricke, C., J. Perry, T. Chapman, and L. Rowe. 2009. Conditional economics of sexual conflict. Biology Letters 5:671-674.

Gavrilets, S. 2000. Rapid evolution of reproductive barriers driven by sexual conflict. Nature 403:886-889.

Gavrilets, S., G. Arnqvist, and U. Friberg. 2001. The evolution of female mate choice by sexual conflict. Proceedings of the Roval Society of London B 268:531-539.

Gioti, A., S. Wigby, B. Wertheim, E. Schuster, P. Martinez, C. J. Pennington, L. Partridge, and T. Chapman. 2012. Sex peptide of D. melanogaster males is a global regulator of reproductive processes in females. Proceedings of the Roval Society B 279:4423-4432.

Guillemaud, T., T. Lenormand, D. Bourguet, C. Chevillon, N. Pasteur, and M. Raymond. 1998. Evolution of resistance in Culex pipiens: allele replacement and changing environment. Evolution 52:443-453.

Hart, M. W., J. M. Sunday, I. Popovic, and C. M. Konrad. 2014. Incipient speciation of sea star populations by adaptive gamete recognition coevolution. Evolution 68:1294-1305.

Hayashi, T. I., M. Vose, and S. Gavrilets. 2007. Genetic differentiation by sexual conflict. Evolution 61:516-529.

Heifetz, Y., O. Lung, E. A. Frongillo, and M. F. Wolfner. 2000. The Drosophila seminal fluid protein Acp26Aa stimulates release of oocytes by the ovary. Current Biology 10:99-102.

Heifetz, Y., L. N. Vandenberg, H. I. Cohn, and M. F. Wolfner. 2005 Two cleavage products of the Drosophila accessory gland protein ovulin can independently induce ovulation. Proceedings of the National Academy of Sciences of the USA 102:743-748.

Holland, B., and W. R. Rice. 1998. Chase-away sexual selection: antagonistic seduction versus resistance. Evolution 52:1-7.

- 1999. Experimental removal of sexual selection reverses intersexual antagonistic coevolution and removes a reproductive load. Proceedings of the National Academv of Sciences of the USA 96:5083-5088.

Imhof, M., B. Harr, G. Brem, and C. Schlotterer. 1998. Multiple mating in wild Drosophila melanogaster revisited by microsatellite analysis. Molecular Ecology 7:915-917.

Innocenti, P., and E. H. Morrow. 2010. The sexually antagonistic genes of Drosophila melanogaster. PLoS Biology 8:e1000335.

Isaac, R. E., C. Li, A. E. Leedale, and A. D. Shirras. 2009. Drosophila male sex peptide inhibits siesta sleep and promotes locomotor activity in the post-mated female. Proceedings of the Roval Society B 277:65-70.

Kennedy, D. R., and A. F. Read. 2017. Why does drug resistance readily evolve but vaccine resistance does not? Proceedings of the Roval Society B 284:20162562.

Khila, A., E. Abouheif, and L. Rowe. 2012. Function, developmental genetics, and fitness consequences of a sexually antagonistic trait. Science 336:585-589.

Kirkpatrick, M., and N. H. Barton. 1997. The strength of indirect selection on female mating preferences. Proceedings of the National Academy of Sciences of the USA 94:1282-1286.

Kokko, H., R. Brooks, J. M. McNamara, and A. I. Houston. 2002. The sexual selection continuum. Proceedings of the Roval Society of London B 269:1333-1340. 
Kokko, H., and D. J. Rankin. 2006. Lonely hearts or sex in the city? density-dependent effects in mating systems. Philosophical Transactions of the Roval Societv B 361:227-386.

Levitan, D. R., and D. L. Ferrell. 2006. Selection on gamete recognition proteins depends on sex, density, and genotype frequency. Science 312:267-269.

Linder, J. E., and W. R. Rice. 2005. Natural selection and genetic variation for female resistance to harm from males. Iournal of Evolutionary Biology 18:568-575.

Liu, H., and E. Kubli. 2003. Sex peptide is the molecular basis of the sperm effect in Drosophila melanogaster. Proceedings of National Academv of Sciences of the USA 100:9929-9933.

Long, T. A. F., R. Montgomerie, and A. K. Chippindale. 2006. Quantifying the gender load: can population crosses reveal interlocus sexual conflict? Philosophical Transactions of the Roval Society B 361:363374

Lonn, E., E. Koskela, T. Mappes, M. Mokkonen, A. M. Sims, and P. C. Watts. 2017. Balancing selection maintains polymorphisms at neurogenetic loci in field experiments. Proceedings of the National Academv of Sciences of the USA 114:3690-3695.

Lung, O., U. Tram, C. M. Finnerty, M. Eipper-Mains, J. M. Kalb, and M. F. Wolfner. 2002. The Drosophila melanogaster seminal fluid protein Acp62F is a protease inhibitor that is toxic upon ectopic expression. Genetics 160:211-224.

Lung, O., and M. F. Wolfner. 2001. Drosophila males transfer antibacterial proteins from their accessory gland and ejaculatory duct to their mates. Journal of Insect Physiology 47:617-622.

Mani, G. S. 1989. Evolution of resistance with sequential application of pesticides in time and space. Proceedings of the Roval Society of London B 238:245-276.

Mokkonen, M., H. Kokko, E. Koskela, J. Lehtonen, T. Mappes, H. Martiskainen, and S. C. Mills. 2011. Negative frequency-dependent selection of sexually antagonistic alleles in Myodes glareolus. $\underline{\text { Sci- }}$ ence 334:972-974.

Moshitzky, P., I. Fleischmann, N. Chaimov, P. Saudan, S. Klauser, E. Kubli, and S. W. Applebaum. 1996. Sex-peptide activates juvenile-hormone biosynthesis in the Drosophila melanogaster corpus allatum. Archives of Insect Biochemistry and Physiology 32:363374.

Mueller, J. L., J. R. Linklater, T. Chapman, and M. F. Wolfner. 2008. Targeted gene deletion and phenotypic analysis of the Drosophila melanogaster seminal fluid protease inhibitor Acp62F. Genetics 178 $1605-1614$

Mueller, J. L., J. L. Page, and M. F. Wolfner. 2007. An ectopic expression screen reveals the protective and toxic effects of Drosophila seminal fluid proteins. Genetics 175:777-783.

Muir, D. A. 1977. Genetic aspects of developing insecticide resistance of malaria vectors. II. Gene flow and control patterns. World Health Organization document WHO/VBC/75.856. World Health Organization, Geneva.

Neubaum, D. M., and M. F. Wolfner. 1999. Mated Drosophila melanogaster females require a seminal fluid protein, Acp36DE, to store sperm efficiently. Genetics 153:845-857.

Paris, M., F. Roux, A. Berard, and X. Reboud. 2008. The effects of the genetic background on herbicide resistance fitness cost and its associated dominance in Arabidopsis thaliana. Heredity 101:499-506.

Parker, G. A. 1979. Sexual selection and sexual conflict. Academic Press, New York.

Parker, G. A., and L. Partridge. 1998. Sexual conflict and speciation. Philosophical Transactions of the Roval Society B 353:261-274.
Partridge, L., and L. D. Hurst. 1998. Sex and conflict. Science 281:20032008.

Peng, J., P. Zipperlen, and E. Kubli. 2005. Drosophila sex-peptide stimulates female innate immune system after mating via the Toll and Imd pathways. Current Biology 15:1690-1694.

Perry, J. C., C. J. Garroway, and L. Rowe. 2017. The role of ecology, neutral processes and antagonistic coevolution in an apparent sexual arms race. Ecology Letters 20:1107-1117.

Ram, K. R., and M. F. Wolfner. 2007a. Seminal influences: Drosophila Acps and the molecular interplay between males and females during reproduction. Integrative and Comparative Biology 47:427-445.

- 2007b. Sustained post-mating response in D. melanogaster requires multiple seminal fluid proteins. PLoS Genetics 3:e221.

.2009. A network of interactions among seminal proteins underlies the long-term postmating response in Drosophila. Proceedings of the National Academy of Sciences of the USA 106:15384-15389.

Read, A. F., S. J. Baigent, C. Powers, L. B. Kgosana, L. Blackwell, L. P. Smith, D. A. Kennedy, S. W. Walkden-Brown, and V. K. Nair. 2015. Imperfect vaccination can enhance the transmission of highly virulent pathogens. PLoS Biology 13:e1002198.

Read, A. F., T. Day, and S. Huijben. 2011. The evolution of drug resistance and the curious orthodoxy of aggressive chemotherapy. Proceedings of the National Academv of Sciences of the USA 108:10871-10877.

Read, A. F., P. A. Lynch, and M. B. Thomas. 2009. How to make evolutionproof insecticides for malaria control. PLoS Biology 7:e1000058.

REX Consortium. 2010. The skill and style to model the evolution of resistance to pesticides and drugs. Evolutionary Applications 3:375-390.

. 2012. Hereogeneity of selection and the evolution of resistance. Trends in Ecology and Evolution 26:110-117.

-2016. Combining selective pressures to enhance the durability of disease resistance genes. Frontiers in Plant Science 7:1916.

Ribeiro, C., and B. J. Dickson. 2010. Sex peptide receptor and neuronal TOR/S6K signaling modulate nutrient balancing in Drosophila. Current Biology 20:1000-1005.

Rice, W. R. 1992. Sexually antagonistic genes - experimental-evidence. Science 256:1436-1439.

. 1996. Sexually antagonistic male adaptation triggered by experimental arrest of female evolution. Nature 381:232-234.

1998. Intergenomic conflict, interlocus antagonistic coevolution and the evolution of reproductive isolation. Pages 261-270 in D. J. Howard and S. H. Berlocher, eds. Endless forms species and speciation. Oxford University Press, Oxford.

2000. Dangerous liasons. Proceedings of the National Academv of Sciences of the USA 97:12953-12955.

Rice, W. R., and B. Holland. 1997. The enemies within: intergenomic conflict, inerlocus contest evolution (ICE), and the intraspecific Red Queen. Behavioural Ecology and Sociobiology 41:1-10.

Rice, W. R., A. D. Stewart, E. H. Morrow, J. E. Linder, N. Orteiza, and P. G. Byrne. 2006. Assessing sexual conflict in the Drosophila melanogaster laboratory model system. Philosophical Transactions of the Roval Society B 361:287-299.

Rodriguez, R. L., and W. A. Snedden. 2004. On the functional design of mate preferences and receiver biases. Animal Behaviour 68:427-432.

Rowe, L., and G. Arnqvist. 2002. Sexually antagonistic coevolution in a mating system: combining experimental and comparative approaches to address evolutionary processes. Evolution 56:754-767.

Rowe, L., E. Cameron, and T. Day. 2003. Detecting sexually antagonistic coevolution with population crosses. Proceedings of the Roval Society of London B 270:2009-2016. 
2005. Escalation, retreat, and female indifference as alternative outcomes of sexually antagonistic coevolution. American Naturalist 165(suppl.):S5-S18.

Rowe, L., and T. Day. 2006. Detecting sexual conflict and sexually antagonistic coevolution. Philosophical Transactions of the Roval Society B 361:277-285.

Ryan, M. J., and M. E. Cummings. 2013. Perceptual biases and mate choice. Annual Review of Ecology and Systematics 44:437-459.

Sakaluk, S. K., R. L. Avery, and C. B. Weddle. 2006. Cryptic sexual conflict in gift-giving insects: chasing the chase away. American Naturalist 167:94-104.

Sirot, L. K., A. Wong, T. Chapman, and M. F. Wolfner. 2014. Sexual conflict and seminal fluid proteins: a dynamic landscape of sexual interactions. Pages 49-72 in W. R. Rice and S. Gavrilets, eds. The genetics and biology of sexual conflict. Cold Spring Harbor, New York

Smith, D. T., N. V. E. Clarke, J. M. Boone, C. Fricke, and T. Chapman. 2017. Sexual conflict over remating interval is modulated by the sex peptide pathway. Proceedings of the Roval Society B 284: 20162394.

Smith, D. T., D. J. Hosken, R. Ffrench-Constant, and N. Wedell. 2009. Variation in sex peptide expression in D. melanogaster. Genetics Research 91:237-242.

Swanson, W. J., C. F. Aquadro, and V. D. Vacquier. 2001a. Polymorphism in abalone fertilisation proteins is consistent with neutral evolution of the egg's receptor for lysin (VERL) and positive Darwinian selection of sperm lysin. Molecular Biology and Evolution 18:376-383.

Swanson, W. J., A. G. Clark, H. M. Waldrip-Dail, M. F. Wolfner, and C. F. Aquadro. 2001b. Evolutionary EST analysis identifies rapidly evolving male reproductive proteins in Drosophila. Proceedings of the National Academy of Sciences of the USA 98:7375-7379.

Swanson, W. J., R. Nielsen, and Q. F. Yang. 2003. Pervasive adaptive evolution in mammalian fertilization proteins. Molecular Biology and Evolution 20:18-20.

Swanson, W. J., and V. D. Vacquier. 1995. Extraordinary divergence and positive Darwinian selection in a fusagenic protein coating the acrosomal process of abalone spermatozoa. Proceedings of the National Academy of Sciences of the USA 92:4957-4961.

1998. Concerted evolution in an egg receptor for a rapidly evolving abalone sperm protein. Science 281:710-712.

. 2002. The rapid evolution of reproductive proteins. Nature Reviews Genetics 3:137-144.

Tram, U., and M. F. Wolfner. 1998. Seminal fluid regulation of female sexual attractiveness in Drosophila melanogaster. Proceedings of the National Academy of Sciences of the USA 95:4051-4054.

- 1999. Male seminal fluid proteins are essential for sperm storage in Drosophila melanogaster. Genetics 153:837-844.

Tregenza, T., N. Wedell, and T. Chapman. 2006. Introduction: sexual conflict: a new paradigm? Philosophical Transactions of the Roval Society B 361:229-234.

West-Eberhard, M. J. 1979. Sexual selection, social competition, and evolution. Proceedings of the American Philosophical Society 123 : $222-234$

Wigby, S., and T. Chapman. 2004. Female resistance to male harm evolves in response to manipulation of sexual conflict. Evolution 58:1028-1037.

- 2005. Sex peptide causes mating costs in female Drosophila melanogaster. Current Biology 15:316-321.

Williams, P. D., T. Day, and E. Cameron. 2005. The evolution of sperm-allocation patterns strategies and the degree of sperm competition. Evolution 59:492-499.

Wolfner, M. F. 1997. Tokens of love: functions and regulation of Drosophila male accessory gland products. Insect Biochemistry and Molecular Biology 27:179-192.

- 2002. The gifts that keep on giving: physiological functions and evolutionary dynamics of male seminal proteins in Drosophila. Heredity 88:85-93.

Wong, A., S. N. Albright, J. D. Giebel, K. R. Ram, S. Q. Ji, A. C. Fiumera, and M. F. Wolfner. 2008. A role for Acp29AB, a predicted seminal fluid lectin, in female sperm storage in Drosophila melanogaster. Genetics 180:921-931.

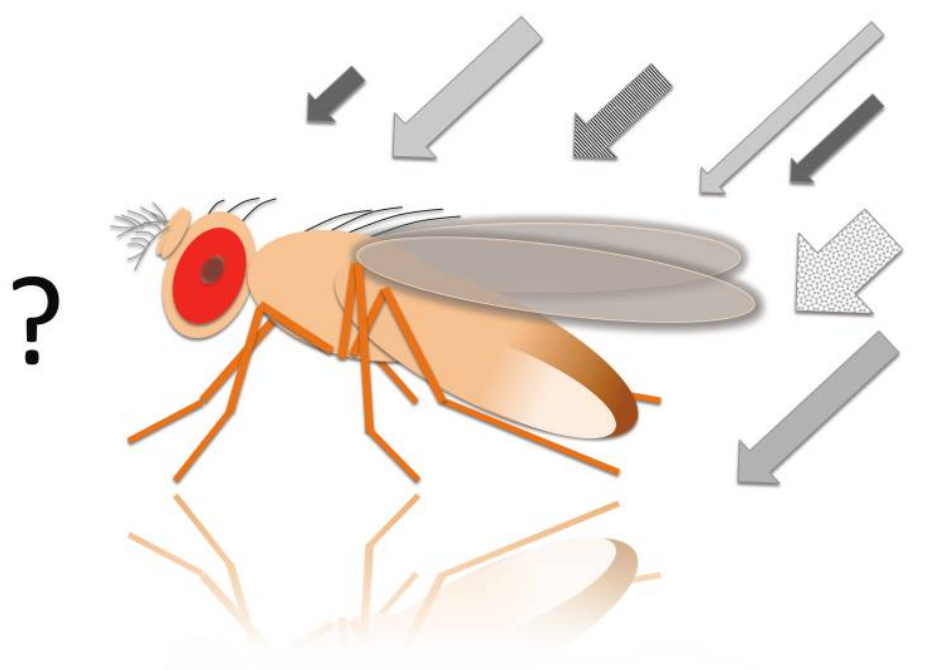

Vice President: Locke Rowe

Females may have to receive many different types of signals and messages from males during mating (represented by the different sizes, shapes, and shades of arrows). Males may use highly complex sets of signals as part of a strategy to make it difficult for females to evolve resistance against those signals. Artwork: Tracey Chapman. 